\title{
Flame propagation across an obstacle: OH-PLIF and 2-D simulations with detailed chemistry
}

\author{
L.R. Boeck ${ }^{\mathrm{a}}$, S. Lapointe ${ }^{\mathrm{a}}$, J. Melguizo-Gavilanes ${ }^{\mathrm{a}}$, G. Ciccarelli ${ }^{\mathrm{b}}$ \\ ${ }^{a}$ Graduate Aerospace Laboratories, California Institute of Technology, Pasadena, CA 91125, USA \\ ${ }^{b}$ Department of Mechanical and Materials Engineering, Queen's University, Kingston, ON K7L 3N6, \\ $C A N$
}

\section{Corresponding Author}

\author{
L.R. Boeck \\ California Institute of Technology \\ 1200 E. California Blvd. MC 205-45 \\ Pasadena, CA 91125 USA \\ E-mail: lbock@caltech.edu
}

\section{Colloquium}

Detonations, explosions and supersonic combustion (8)

\author{
Word count \\ Method: 2 \\ Full pages: 6 pages $\mathrm{x} 900$ words/page $=5400$ words \\ Partial pages: 1 page $-45 \mathrm{~mm} \times 4.4$ words $/ \mathrm{mm}=189$ words \\ 1 page $-75 \mathrm{~mm} \times 2.2$ words $/ \mathrm{mm}=165$ words \\ Revisions: 109 words \\ TOTAL length of paper: $5754+109=5863$ words \\ Main text: $3821+109$ words
}

Figures: (1) 85 words; (2) 65 words; (3) 170 words; (4) 360 words; (5) 244 words; (6) 191 words; (7) 170

$$
\begin{aligned}
& \text { words; (8) } 170 \text { words } \\
& \text { Tables: (1) } 138 \text { words } \\
& \text { References: } 340 \text { words }
\end{aligned}
$$

Supplemental material is available

The authors will not pay color reproduction charges 


\begin{abstract}
Flame propagation across a single obstacle inside a closed square channel is studied experimentally and numerically using a stoichiometric $\mathrm{H}_{2} / \mathrm{O}_{2}$ mixture at initial conditions $15 \mathrm{kPa}$ and $300 \mathrm{~K}$. The $50 \%$ blockage obstacle consists of a pair of fence-type obstacles mounted on the top and bottom walls of the channel. Direct optical visualization was performed using single-image measurement of the planar laser-induced fluorescence of the OH radical (OH-PLIF) and simultaneous high-speed schlieren video to study the flame topology and the flame tip velocity along the channel streamwise axis, respectively. The OH-PLIF images provide a novel level of detail and permit a thorough evaluation of the simulation accuracy. The flame tip accelerates to a peak velocity of $590 \mathrm{~m} / \mathrm{s}$ just downstream of the obstacle followed by a deceleration and subsequent re-acceleration. The unburnt gas flow ahead of the flame is subsonic at all times. The flame does not show any signs of diffusive-thermal instability. Vortex-flame interactions in the recirculation zones downstream of the obstacle wrinkle the flame. The numerical simulations, based on solving the 2-D compressible reactive Navier-Stokes equations with detailed chemistry, predict the flame tip velocity accurately. However, differences in flame topology are observed, specifically, wrinkling is over-estimated. The over-prediction of flame wrinkling suggests a lower dissipation rate in the numerical simulations than in reality, which could be a consequence of neglecting the third channel dimension. Conditional means of the fuel consumption rate are similar to the consumption rates of 1-D unstretched laminar flames at all times. The increase in pressure during flame propagation causes an increase in fuel consumption rate which needs to be accounted for in simplified modeling approaches.
\end{abstract}

Keywords: Flame acceleration; OH-PLIF; Numerical simulations; Detailed chemistry

\title{
1. Introduction
}

Studies on flame propagation in tubes date back to the pioneering work of Berthelot and Vieille [1] and Mallard and Le Chatelier [2]. Flames in a closed unobstructed tube were observed to accelerate and undergo transition to detonation. Chapman and Wheeler [3] reported that obstacles in a tube can significantly promote flame acceleration which was visualized by streak photography. Strong acceleration was attributed to the increase in the burning rate due to turbulence generated in the wake of obstacles. Moen et al. [4] highlighted the positive feedback between the flame and flow generated in the unburnt mixture ahead of it. Vortices downstream of obstacles "fold" the flame and thus increase the flame surface area and the volumetric burning rate. Visualization of the flow around obstacles by Johansen and Ciccarelli [5] identified the importance of the flame interaction with the vortices in obstacle wakes and turbulent shear layers emanating from obstacle edges. More recently, the role of shock-flame interactions in flame acceleration was characterized by Ciccarelli et al. [6] by means of highly time-resolved schlieren imaging. This technique, being the most commonly used for direct visualization of explosion dynamics on laboratory scale, delivered detailed flame velocity measurements. Line-of-sight integration inherent to the schlieren technique, complicates the analysis of the flame topology. The review by Ciccarelli and Dorofeev [7] offers a wide perspective on the current state of knowledge on flame propagation in tubes. 
Numerical simulations of flame propagation in tubes have provided useful insight. Large Eddy Simulation (LES) coupled with simplified chemistry have been used to model the phenomenon. Reasonable agreement with experiments in terms of global properties such as flame velocity and overpressure has been demonstrated; flame topology at scales resolved by the LES are oftentimes reproduced well [8, 9]. To study flame and flow structure in greater detail all relevant physical scales present in the system need to be resolved. However, direct numerical simulations with detailed chemistry remain computationally expensive. A typical compromise when performing detailed simulations is to neglect one of the physical dimensions (i.e. 2-D vs. 3-D) or to use simplified chemistry. To date, the use of detailed chemistry and transport in channel flame propagation cases has been limited to $\mathrm{H}_{2}$ as a fuel. Two- and three-dimensional simulations performed by Gamezo et al. [10] and Kessler et al. [11] using simplified chemistry (one-step Arrhenius calibrated to match laminar flame and detonation properties) successfully reproduced qualitatively, and in some cases also quantitatively, experimental observations in stoichiometric $\mathrm{H}_{2}$ /air and $\mathrm{CH}_{4} /$ air sytems. Detailed chemistry was used in 2-D simulations of deflagration-to-detonation transition in an unobstructed $2 \mathrm{~mm}$ diameter tube filled with $\mathrm{H}_{2} / \mathrm{O}_{2}$ by Dziemińska and Hayashi [12]. Liberman $e t$ $a l$. [13] and Ivanov et al. [14] recently presented results from 3-D simulations using detailed chemistry in $\mathrm{H}_{2} / \mathrm{O}_{2}$. They investigated flame acceleration and mechanisms of transition to detonation in an unobstructed square channel with non-slip walls. Comparison of their results with previous experimental work in a similar geometry was in reasonable qualitative agreement. In contrast with simulations of unobstructed channels, Gamezo et al. [10] argued that numerical predictions of channels with fence-type obstacles show only minor differences in global behavior (e.g. flame tip velocity) when the channel spanwise dimension is neglected. The authors propose that fence-type obstacles generate essentially two-dimensional shear layers.

In the present study, we compare experimental observations and numerical predictions of the flame propagation inside a closed square cross-section channel with a single obstacle, filled with a stoichiometric $\mathrm{H}_{2} / \mathrm{O}_{2}$ mixture at initial conditions $15 \mathrm{kPa}$ and $300 \mathrm{~K}$. We use a well characterized experiment [5, 6], equipped with OH-PLIF diagnostics to obtain high-resolution images of the flame topology at a novel quality, and the widely validated NGA code [15] to perform 2-D simulations, based on solving the compressible reactive Navier-Stokes equations with detailed chemistry. The analysis focuses on a one-to-one comparison of the simulations with experimental results, especially the OH-PLIF, since these represent true 2-D images of the flame in the OH-PLIF laser plane (unlike line-of-sight integrated schlieren images) and thus allow for a very critical view of the simulations. Vortex-flame interactions and effects of differential diffusion are discussed. 


\section{Experimental and numerical methodology}

\subsection{Experimental setup}

Experiments were performed in an entirely closed channel, see Fig. 1. The cross-section and length were $0.0762 \times 0.0762 \mathrm{~m}(3 \times 3 \mathrm{in})$ and $0.4953 \mathrm{~m}(29.5 \mathrm{in})$, respectively. A single obstacle was located at $x=0.1524 \mathrm{~m}$ (6 in). The obstacle consisted of an upper and lower wall mounted fence-type obstacle, each with a height of $0.0191 \mathrm{~m}(0.75 \mathrm{in})$ and a thickness of $0.0127 \mathrm{~m}$ (0.5 in), extending across the channel spanwise dimension. Sidewall windows permitted optical access. Mixtures were prepared in a separate mixing vessel using the method of partial pressures and filled into the previously evacuated channel. A spark plug centered at the channel end wall $(x=0 \mathrm{~m})$ ignited the mixture.

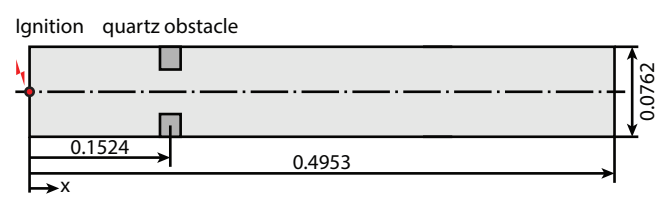

Figure 1: Schematic of the experimental setup, side view. Dimensions are given in $\mathrm{m}$.

\subsection{Measurement techniques}

Two optical measurement techniques were applied simultaneously: Single-image OH-PLIF (Planar LaserInduced Fluorescence) and $75 \mathrm{kfps}$ schlieren imaging. The OH-PLIF system consisted of a Spectra Physics Quanta Ray PIV 400 pumplaser, a Sirah Precision Scan dye laser with Rhodamie 6G as a dye and a Princeton Instruments PI-MAX image-intensified camera equipped with a UV-Nikkor $105 \mathrm{~mm} \mathrm{f} / 4.5$ camera lens with an interference filter, $312 \mathrm{~nm}$ central wavelength, $F W H M=24 \mathrm{~nm}$. OH radicals were excited at their $Q_{1}(6)$ transition, $\lambda \approx 282.9 \mathrm{~nm}$. The laser beam was unidirectionally expanded with a $f=-30 \mathrm{~mm}$ cylindrical lens and collimated with a $f=500 \mathrm{~mm}$ spherical lens. The light sheet entered the channel through a vertical quartz window in the channel end plate $(x=0.4953 \mathrm{~m})$ on the spanwise centerline, covering the entire channel height. The obstacle was made from quartz glass capable of transmitting the OH-PLIF laser light sheet. The schlieren setup consisted of two concave mirrors, $f=3.048 \mathrm{~m}$, two planar turning mirrors, a constant white Xenon arc light source and a Photron SA-5 camera. The schlieren camera was directly triggered off the ignition signal. One OH-PLIF image was captured per experiment. Varying the delay time between the ignition signal and capturing the OH-PLIF image permitted generating a coherent OH-PLIF sequence.

Figure 2 illustrates the great benefit of OH-PLIF. One OH-PLIF (left) and a schlieren image (right) taken simultaneously in one exemplary experiment are shown. While OH-PLIF resolves flame wrinkling due to vortexflame interactions downstream of the obstacle in great detail, the analysis of flame topology based on the lineof-sight integrated schlieren image would be susceptible to misinterpretation. 


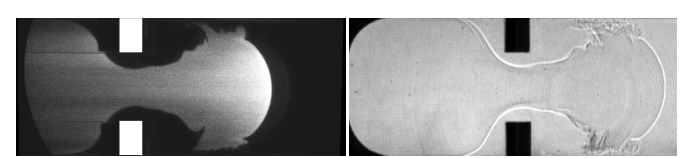

Figure 2: Left: OH-PLIF image, see frame 8 in Fig. 4. Right: schlieren image taken at the same time.

\subsection{Computational setup - Governing equations}

The flame propagation and acceleration are modeled by solving the compressible reactive Navier-Stokes equations in 2-D [16]. Conservation of mass, momentum, species transport, and total energy read

$$
\begin{gathered}
\frac{\partial \rho}{\partial t}+\nabla \cdot(\rho \mathbf{u})=0 \\
\frac{\partial \rho \mathbf{u}}{\partial t}+\nabla \cdot(\rho \mathbf{u} \otimes \mathbf{u})=-\nabla p+\nabla \cdot \tau \\
\frac{\partial \rho Y_{i}}{\partial t}+\nabla \cdot\left(\rho \mathbf{u} Y_{i}\right)=-\nabla \cdot \mathbf{j}_{\mathbf{i}}+\rho \dot{\omega}_{i} \\
\frac{\partial \rho e_{t}}{\partial t}+\nabla \cdot\left(\mathbf{u}\left(\rho e_{t}+p\right)\right)=\nabla \cdot \mathbf{j}_{\mathbf{q}}+\nabla \cdot(\tau \cdot \mathbf{u})
\end{gathered}
$$

In these equations, $\rho$ is the fluid density, $\mathbf{u}$ is the velocity vector, $p$ is the pressure, $\tau$ is the viscous stress tensor, $Y_{i}$ is the species mass fraction, $\dot{\omega}_{i}$ is the species production rate, $e_{t}$ is the total energy, $\mathbf{j}_{\mathbf{i}}$ is the species diffusion mass flux, $\mathbf{j}_{\mathbf{q}}$ is the heat flux, and $\dot{\omega}_{i}$ is the production/consumption rate of species. The species diffusion term is defined as:

$$
\mathbf{j}_{\mathbf{i}}=-\rho D_{i, m} \frac{Y_{i}}{X_{i}} \nabla X_{i}-\rho Y_{i} \mathbf{u}_{\mathbf{c}}
$$

where $X_{i}$ is the species molar fraction, $D_{i, m}$ is the diffusion coefficient of species $i$ into the mixture, and $\mathbf{u}_{\mathbf{c}}$ is the correction velocity to ensure conservation of mass when using a mixture-averaged formulation:

$$
\mathbf{u}_{\mathbf{c}}=-\sum_{i} D_{i, m} \frac{Y_{i}}{X_{i}} \nabla X_{i}
$$

The heat flux term is given by:

$$
\mathbf{j}_{\mathbf{q}}=\lambda \nabla T+\sum_{i=1}^{N} h_{i} \mathbf{j}_{\mathbf{i}}
$$

where $\lambda$ is the mixture thermal conductivity, $T$ is the temperature, and $h_{i}$ is the species enthalpy. The fluid is treated as a perfect gas with the equation of state

$$
p=\rho R T \sum_{i} \frac{Y_{i}}{W_{i}}
$$

where $R$ is the universal gas constant, and $W_{i}$ is the species molecular weight. Since the system is solved in terms of the conserved variables, the temperature is obtained through an implicit equation. 
The governing equations are solved using a compressible version of the energy conservative, finite difference code NGA [15]. The simulations use a second-order accurate spatial discretization on a structured, staggered, cartesian grid. A fourth order accurate, explicit Runge-Kutta time integration is used. A preconditioning strategy based on a diagonal approximation of the chemical Jacobian [17] is used to integrate the chemical source terms. The third-order Bounded QUICK scheme, BQUICK [18], is used as the transport scheme of the species and temperature/energy to ensure that the scalars remain within their physical bounds.

\subsection{Simulation parameters}

Two-dimensional simulations are performed with both a mixture-averaged formulation for diffusion coefficients $(L e \neq 1)$ and constant unity Lewis numbers $(L e=1)$. This allows to investigate whether multidimensional differential diffusion affects the flame. Table 1 lists the simulation parameters.

\begin{tabular}{|c|c|c|}
\hline Case & \multicolumn{2}{|c|}{$\begin{array}{c}\mathbf{H}_{2} / \mathbf{O}_{2} \\
\mathbf{L e} \neq \mathbf{L} \mathbf{e}=\mathbf{1}\end{array}$} \\
\hline$p_{0}(\mathrm{kPa})$ & \multicolumn{2}{|c|}{15} \\
\hline$T_{u}(\mathrm{~K})$ & \multicolumn{2}{|c|}{300} \\
\hline$\phi$ & \multicolumn{2}{|c|}{1.0} \\
\hline$S_{L}(\mathrm{~m} / \mathrm{s})$ & 7.05 & 4.88 \\
\hline$l_{F}(\mathrm{~mm})$ & 2.78 & 2.10 \\
\hline$T_{\text {peak }}$ & 1375 & 1370 \\
\hline$\Delta x=\Delta y(\mathrm{~m})$ & \multicolumn{2}{|c|}{$9.3 \times 10^{-5}$} \\
\hline Grid & \multicolumn{2}{|c|}{$5345 \times 822$} \\
\hline
\end{tabular}

Table 1: Parameters of the simulations and flame properties. $p_{0}$ is the initial pressure, $T_{u}$ is the initial unburnt temperature, $\phi$ is the equivalence ratio, $S_{L}$ is the laminar flame speed, $l_{F}=\left(T_{b}-T_{u}\right) /|\nabla T|_{\max }$ is the laminar flame thickness, $\Delta x=\Delta y$ is the grid spacing, and $T_{\text {peak }}$ is the temperature of peak fuel consumption in the laminar flame.

The dimensions of the computational domain are identical to the experimental channel (Fig. 1). A uniform grid spacing of $\Delta x=\Delta y=9.3 \cdot 10^{-5} m \approx l_{F} / 30$ is used. This corresponds to approximately 20 cells per $\mathrm{OH}$ reaction layer. Grid independence with respect to flame tip velocity and chemical source terms was assessed. However, while flame scales are fully resolved, small flow scales present at these high Re numbers may not be captured with the current resolution. Regions of turbulent flow in the shear layers behind the obstacle are not fully resolved in the present two-dimensional simulations. No-slip boundary conditions are applied on the velocity components and zero gradient boundary conditions are specified for the transported scalars.

To mimic the experimental ignition, simulations are initialized with a hemi-cylindrical flame kernel of radius $2 \mathrm{~mm}$ centered at the channel end wall (see Fig. 1). The initial density, temperature, and species mass fractions of this flame kernel are taken from the 1-D laminar flame.

\subsection{Chemical and transport models}

The hydrogen mechanism of Hong et al. [19] consisting of 10 species and 31 reactions is used. The species viscosities $\mu_{i}$ are computed from standard gas kinetic theory [20] and the mixture-averaged viscosity is calculated using a modified form of Wilke's formula [21,22]. The species thermal conductivities, $\lambda_{i}$, are computed 
using Eucken's formula [23] and the mixture-averaged thermal conductivity $\lambda$ is obtained following Mathur et $a l$. [24]. Species diffusivities are computed using mixture-averaged diffusion coefficients [25]. Soret and Dufour effects are neglected.

\section{Results and Discussion}

\subsection{Flame tip velocity}

Experimentally, the flame tip velocity along the channel centerline is obtained from the high-speed schlieren video (made available as supplemental material). In the simulations, the location of the $1500 \mathrm{~K}$ isotherm is tracked along the channel centerline. Figure 3 compares the experimental and simulation flame tip velocities. Results of three different experiments are plotted together with the numerical predictions with $L e \neq 1$ and $L e=1$. The simulations qualitatively reproduce the experimentally observed initial acceleration across the obstacle, the location of the velocity peak (slightly downstream of the obstacle), the deceleration downstream of the obstacle and the subsequent secondary acceleration. The initial acceleration and deceleration is caused by the contraction and expansion of the unburnt gas through the obstacle opening. The secondary acceleration is associated with the rapid turbulent combustion in the recirculation zones downstream of the obstacle. Quantitatively, the $L e \neq 1$ formulation is in very good agreement with the experimental results. The slight underprediction of the flame tip peak velocity is likely an effect of neglecting the third channel dimension, as pointed out by Gamezo et al. [10]. Note that the $L e=1$ simulation result is not expected to reproduce the experiment but serves as a base for later discussion.

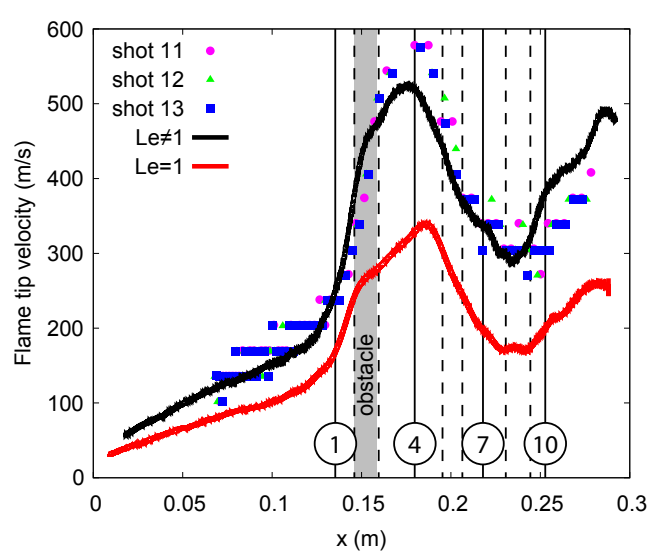

Figure 3: Flame tip velocity plotted against position in the channel. Obstacle is filled in grey. Vertical solid (dashed) lines indicate flame tip positions for frames 1, 4, 7, $10(2,3,5,6,8,9)$ in Fig. 4.

\subsection{Flame topology}

Figure 4 shows OH-PLIF images (left), and the numerical temperature fields (right) obtained with $L e \neq 1$. The experimental sequence is reconstructed from ten different experiments with a difference in trigger delay 


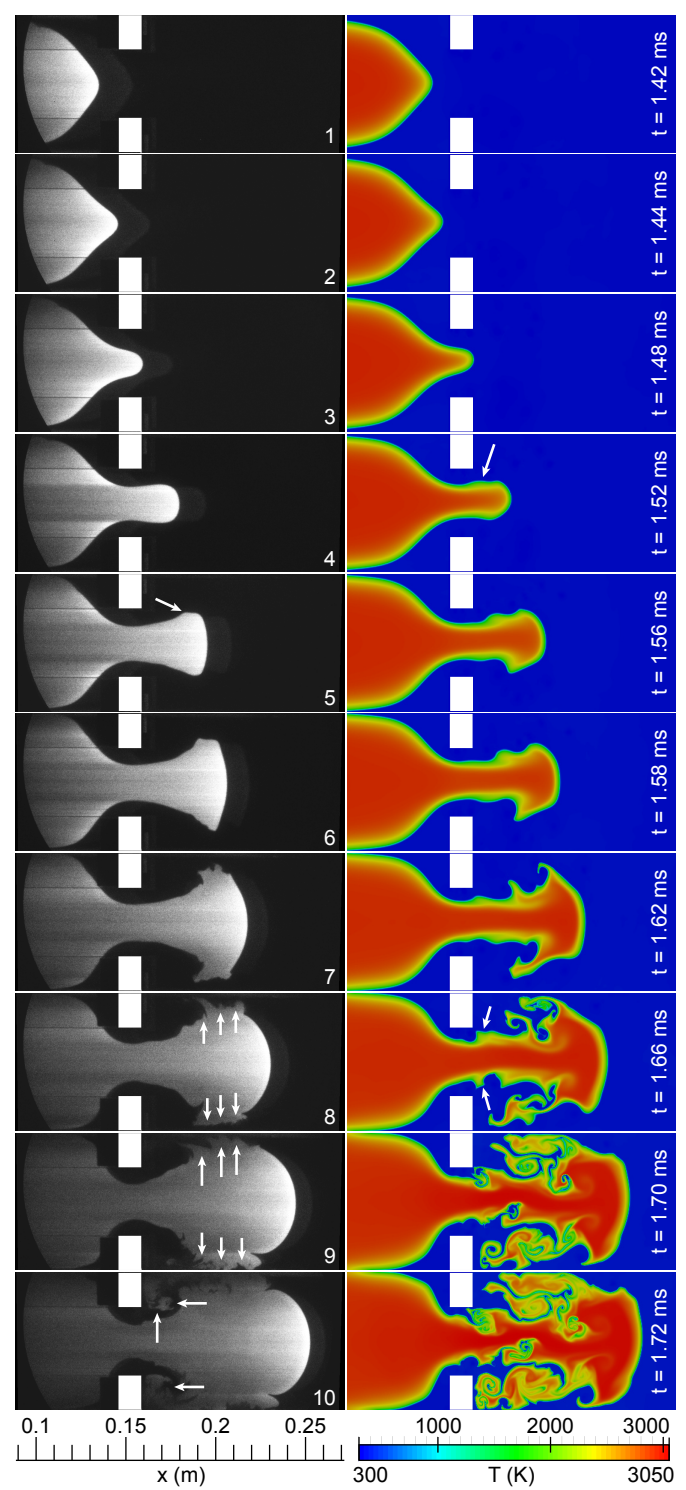

Figure 4: Left: experimental OH-PLIF images from ten experiments. Right: temperature fields from simulations.

time between the images of $\Delta t=35 \mu \mathrm{s}$. The numerical fields are selected to match the experimental axial flame tip locations (indicated in Fig. 3, vertical lines); the times reported are the corresponding simulation times. The description of the flame evolution is divided into two phases according to the observed flame topology in the $\mathrm{OH}$ PLIF images: (1) flame propagation upstream and past the obstacle showing a smooth (laminar) flame surface (frames 1-4) and (2) frames 5-10 with flame tip locations further downstream of the obstacle and a progressively wrinkled flame front.

(1) Frames 1-4 show entirely smooth (laminar) flame fronts. No cellular structure of the flame front is observed which suggests diffusive-thermal stability. The absence of perturbations also shows that no significant turbulent motion is present immediately ahead of the flame front. The flame tip stretches as it approaches (frame 2 ) and passes (frames 3 and 4 ) through the obstacle opening. The peak flame tip velocity of $v \approx 590 \mathrm{~m} / \mathrm{s}$, see Fig. 3, is reached in frame 4. Good agreement in terms of flame topology is observed between simulations and experiments. 
(2) Frame 5 in the OH-PLIF sequence shows the first signs of the interaction of vortices being shed off the obstacle edges and the flame, resulting in a slight local deformation of the flame (see arrow in frame 5). In contrast to diffusive-thermally unstable mixtures like lean $\mathrm{H}_{2}$ /air, flame perturbations in the OH-PLIF images can be unambiguously attributed to the interaction of the flame with the flow (and in particular with vortices). The location of vortex-flame interaction is determined by the trajectory of vortices being convected downstream and the lateral expansion of the flame tip as observed in frame 5. Vorticity fields presented in Sec. 3.3 reveal further details. Frame 6 shows a progression of the flame wrinkling and frame 7 represents the beginning of flame convection into the recirculation zones downstream of the obstacle (slightly asymmetrical with earlier flame convection into the lower obstacle recirculation zone). In frames 8 and 9, small-scale wrinkling is observed where the flame approaches the upper and lower channel walls (see arrows). Frame 9 corresponds to the local minimum in the flame tip velocity $(v \approx 300 \mathrm{~m} / \mathrm{s}, x \approx 0.245 \mathrm{~m})$, observed in Fig. 3 . Frame 10 shows the consumption of unburnt mixture inside the recirculation zones. Flame wrinkling at scales down to about $1 \mathrm{~mm}$ is observed in the recirculation zones and near-wall regions downstream of the obstacle. Note that smaller structures may exist but cannot be resolved optically due to image intensifier noise and strong absorption of the laser light resulting in a low signal in the recirculation regions. The leading flame tip remains smooth (laminar) at all times also in phase (2).

The OH-PLIF technique is particularly beneficial to reveal similarities and differences between experiments and simulations in phase (2). As soon as flame wrinkling occurs, the schlieren technique would not deliver a clear picture of the flame topology as demonstrated in Fig. 2. The simulations reproduce the general appearance of the flame in phase (2), i.e., the laminar leading flame tip and the wrinkled flame in the recirculation zones. However, the flame becomes perturbed earlier in the simulations than in the experiments (see arrow at $t=1.52 \mathrm{~ms}$ ). Asymmetry is visible with an earlier flame convection into the lower recirculation zone (e.g. $t=1.56 \mathrm{~ms}$ and $t=1.62 \mathrm{~ms})$. In contrast with the experiments, some minor wrinkling of the flame is observed near the channel centerline at the obstacle opening (see arrows, $t=1.66 \mathrm{~ms}$ ). The one-to-one comparison of OH-PLIF images and computed temperature fields demonstrates that reproducing the exact flame topology is challenging, and the OH-PLIF experiments represent a demanding validation case. Comparison of flame tip velocities did not reveal these inaccuracies. The overprediction of flame wrinkling suggests a lower dissipation rate in the numerical simulations than in reality, which could be a consequence of neglecting the third channel dimension.

\subsection{2-D vorticity and velocity fields}

Vorticity and velocity fields from the numerical simulations are presented in this section to deepen the analysis of the flame propagation process and to trace back the observed differences between experiments and simulations to the transient unburnt gas flow development. Figure 5 shows the magnitude of vorticity in logarithmic scale (left), and the magnitude of velocity with a superimposed flame isocontour ( $T=1500 \mathrm{~K})$ (right) 


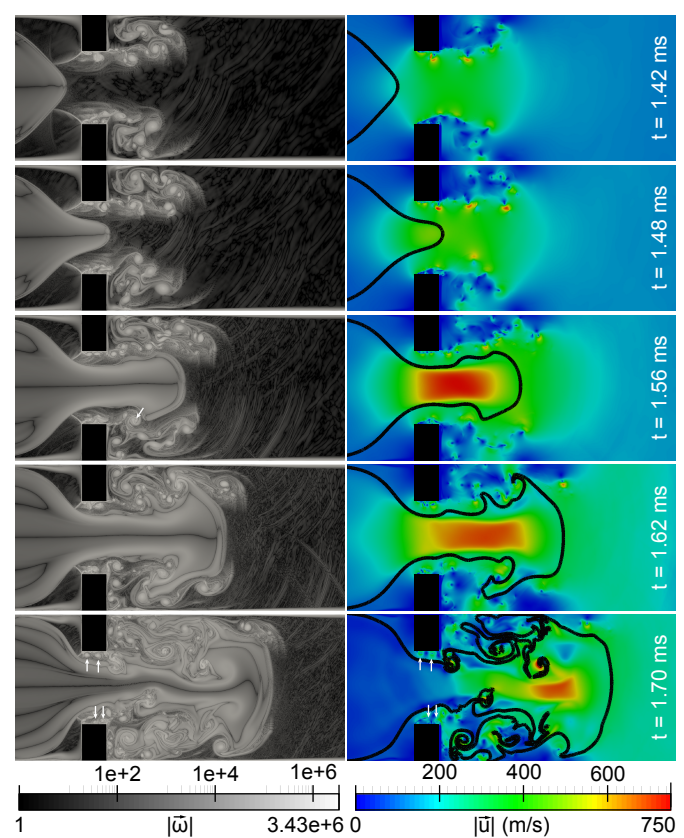

Figure 5: Left: magnitude of vorticity. Right: magnitude of velocity with flame front contour $(T=1500 \mathrm{~K})$, black line.

at five different instances during the simulations.

At $t=1.42 \mathrm{~ms}$, the vorticity field shows the unburnt gas flow through the obstacle opening induced by the thermal expansion of the combustion products. Three important features are worth mentioning: (1) Vortex shedding off the obstacle corners (smaller vortices) with a different shedding frequency and size of vortices at the upper/lower obstacle. This flow-field asymmetry will translate into the flame asymmetry observed at later times. Since the initial conditions in the simulation are symmetric and no initial perturbations are added, the observed asymmetry suggests that the accumulation of numerical error across the simulated time is sufficient to alter the flow field and, eventually, the flame topology. (2) Larger scale vortices present immediately downstream of the obstacle forming the upper and lower obstacle recirculation zones. (3) Essentially irrotational flow in a region along the channel horizontal centerline ahead of the flame. A flame propagating in this region will not be wrinkled. The velocity field at $t=1.42 \mathrm{~ms}$ shows an unburnt gas velocity of about $400 \mathrm{~m} / \mathrm{s}$ in the obstacle opening, the flow is thus subsonic with respect to the unburnt mixture sound speed (sound speed at initial conditions is $540 \mathrm{~m} / \mathrm{s}$ ). Also at later times, the flow remains subsonic in the entire domain.

Flame wrinkling at later times can be attributed to vortex-flame interactions well visible in the vorticity fields. At $t=1.56 \mathrm{~ms}$, a vortex perturbs the flame (see arrow). This one vortex is the main cause for the observed flame asymmetry. The leading flame tip remains laminar at all times because the flame tip propagates preferentially along the center of the channel, bounded by the vortices shed off the obstacle edges, but does not interact with them.

The high-velocity core region that extends along the center of the channel at $t=1.56 \mathrm{~ms}$ in the burnt gas is the combined transient effect of the acceleration of the flow due to the area reduction in the obstacle, and the 
thermal expansion of the combustion products across the flame front. The high-velocity core region is shortlived, the flow in the obstacle opening is brought to rest shortly after, between $t=1.62 \mathrm{~ms}$ and $t=1.70 \mathrm{~ms}$. Small vortices are continuously generated at the obstacle upstream corners (see white arrows, $t=1.70 \mathrm{~ms}$ ). This prevents the flame from contacting the obstacle inner-edge, even when the flame tip is well past the obstacle.

\subsection{Chemical source terms}

Conditional means of the fuel consumption rate on temperature are evaluated to determine the influence of vortex-flame interactions and multi-dimensional differential diffusion effects on the chemical source terms. Figure 6 presents the conditional means of the fuel consumption rate for the non-unity Lewis number cases at three instants before $(1.2 \mathrm{~ms})$, during $(1.48 \mathrm{~ms})$, and after $(1.7 \mathrm{~ms})$ passage past the obstacle. All curves are normalized by the peak burning rate for a 1-D laminar flame at the initial pressure of $15 \mathrm{kPa}$. The burning rate increases in time due to the increase in pressure as the flame propagates through the closed channel. The mean burning rate follows closely the corresponding laminar flame computed at the same pressure. In this diffusivethermally stable flame, there are no multi-dimensional differential diffusion effects influencing the mean burning rate.

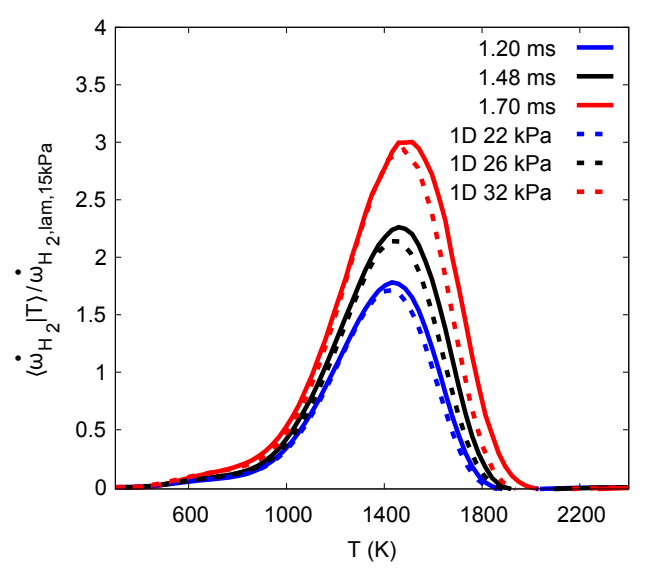

Figure 6: Conditional means of the fuel consumption rate for the non-unity Le number case at three instants. Solid lines represent conditional means from the 2-D simulations. Dashed lines represent solutions from 1-D laminar flames at the corresponding pressure. All curves are normalized by the peak burning rate in the non-unity Le 1-D laminar flame at $15 \mathrm{kPa}$.

A similar behavior is observed for the unity Lewis number flame, as shown in Fig. 7. The conditional means are taken at three instants $(1.7,2.1$, and $2.5 \mathrm{~ms})$ corresponding to the same flame tip locations in the channel as the non-unity Lewis number flame. Again, an increase in the burning rate due to the increased pressure is observed, following the laminar behavior. This indicates that both flames locally behave like one-dimensional laminar flames when the pressure dependence is accounted for.

This is further exemplified by normalizing the simulated flame tip velocity profiles, Fig. 3, with their respective 1-D laminar flame speed (Tab. 1) as shown in Fig. 8. Since the volumetric burning rate is well described by the laminar burning velocity, this normalization collapses the curves. 


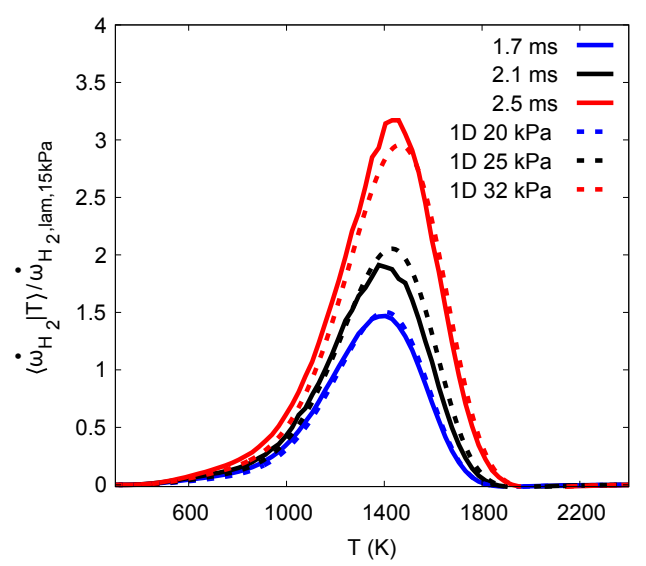

Figure 7: Conditional means of the fuel consumption rate for the unity Le number case at three instants. Solid lines represent conditional means from the 2-D simulations. Dashed lines represent solutions from 1-D laminar flames at the corresponding pressure. All curves are normalized by the peak burning rate in the unity Le 1-D laminar flame at $15 \mathrm{kPa}$.

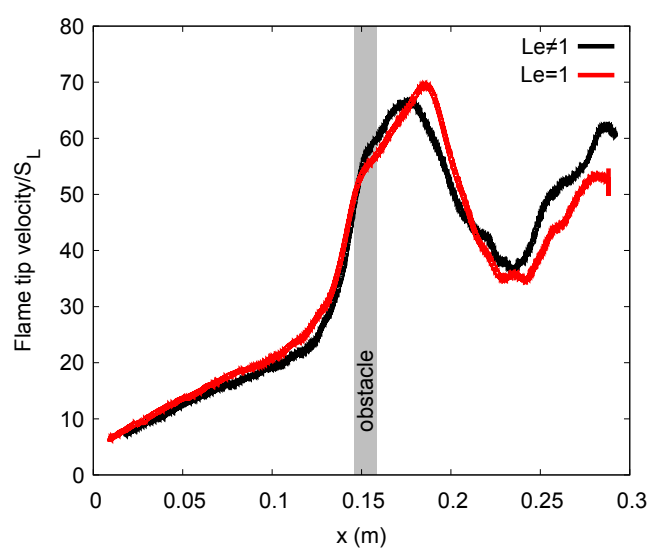

Figure 8: Flame tip velocity, normalized by the respective 1-D laminar flame speed, plotted against position in the channel.

These observations have implications for modeling. Mean quantities $\left(Y_{i}, \dot{\omega}_{i}\right.$, etc.) could be adequately modeled without detailed chemistry. A tabulated chemistry approach based on laminar flames would be sufficient. However, the pressure (and temperature) dependence of the chemical source terms must be taken into account in order to adequately predict the burning rate.

\section{Conclusion}

In the present study the flame propagation past a single obstacle in a closed square channel filled with a stoichiometric $\mathrm{H}_{2} / \mathrm{O}_{2}$ mixture at initial conditions $15 \mathrm{kPa}$ and $300 \mathrm{~K}$ was investigated experimentally and numerically. The OH-PLIF technique resolved the flame topology at a novel level of detail and permitted a critical interpretation of the 2-D simulation results. Comparing simulations against such detailed experimental data reveals deviations that may be overlooked when using more global validation data like flame tip velocity or (line-of-sight integrated) schlieren images. While the simulations reproduced experimental flame tip velocities accurately, vortex-flame interactions downstream of the obstacle were over-estimated, leading to stronger flame 
wrinkling in the simulations. This may be due to an insufficiently low dissipation rate as a result of neglecting the third channel dimension. Analysis of the flow field showed that the unburnt gas flow ahead of the flame remained subsonic at all times. The flame was further characterized by computing the conditional means of the fuel consumption rate, which were observed to be increased by pressure but not significantly influenced by vortex-flame interactions. Multi-dimensional effects of differential diffusion did not occur. This suggests that a diffusive-thermally stable flame propagating past an obstacle in subsonic flow could be modeled accurately without resolving the internal flame structure. However, it is necessary to account for the pressure depencence of the laminar burning velocity. Simulations following a simplified approach (e.g. one-step or tabulated chemistry) will be the topic of future work.

\section{Acknowledgements}

The authors are grateful to the National Research Council Canada for the loan of the intensified camera and UV lens, to Dr. Matthew Johnson from Carleton University for the loan of the lasers and optics, and to Adam Steinberg for the loan of the $\mathrm{OH}$ narrow band filter. This work used the Extreme Science and Engineering Discovery Environment (XSEDE), which is supported by National Science Foundation grant number ACI-1053575, and J. Melguizo-Gavilanes was supported by NSERC Postdoctoral Fellowship Program.

\section{References}

[1] M. Berthelot, P. Vieille, CR Acad. Sci., Paris 93 (1881) 18-22.

[2] E. Mallard, H. Le Chatelier, CR Acad. Sci., Paris 93 (1881) 145-148.

[3] W. R. Chapman, R. V. Wheeler, J. Chem. Soc. 129 (1926) 2139-2147.

[4] I. Moen, M. Donato, R. Knystautas, J. Lee, Combust. Flame 39 (1) (1980) 21-32.

[5] C. T. Johansen, G. Ciccarelli, Combust. Flame 156 (2) (2009) 405-416.

[6] G. Ciccarelli, C. T. Johansen, M. Parravani, Combust. Flame 157 (11) (2010) 2125-2136.

[7] G. Ciccarelli, S. Dorofeev, Prog. Energ. Combust. 34 (4) (2008) 499-550.

[8] A. Masri, S. Ibrahim, B. Cadwallader, Exp. Therm. Fluid Sci. 30 (7) (2006) 687-702.

[9] V. Di Sarli, A. Di Benedetto, G. Russo, S. Jarvis, E. J. Long, G. K. Hargrave, Flow Turbul. Combust. 83 (2) (2009) 227-250.

[10] V. N. Gamezo, T. Ogawa, E. S. Oran, Proc. Combust. Inst. 31 (2) (2007) 2463-2471.

[11] D. Kessler, V. Gamezo, E. Oran, Combust. Flame 157 (11) (2010) 2063-2077. 
[12] E. Dziemńska, A. K. Hayashi, Int. J. Hydrogen Energ. 38 (10) (2013) 4185-4193.

[13] M. Liberman, M. Ivanov, A. Kiverin, M. Kuznetsov, A. Chukalovsky, T. Rakhimova, Acta Astronaut. 67 (7) (2010) 688-701.

[14] M. Ivanov, A. Kiverin, I. Yakovenko, M. A. Liberman, Int. J. Hydrogen Energ. 38 (36) (2013) 1642716440.

[15] O. Desjardins, G. Blanquart, G. Balarac, H. Pitsch, J. Comp. Phys. 227 (15) (2008) 7125-7159.

[16] T. Poinsot, D. Veynante, Theoretical and numerical combustion, RT Edwards, Inc., 2005.

[17] B. Savard, Y. Xuan, B. Bobbitt, G. Blanquart, J. Comp. Phys. 295 (2015) 740-769.

[18] M. Herrmann, G. Blanquart, V. Raman, AIAA J. 44 (2006) 2879-2886.

[19] Z. Hong, D. Davidson, R. Hanson, Combust. Flame 158 (4) (2011) 633-644.

[20] O. Hirschfelder, C. Curtiss, R. Bird, Molecular Theory of Gases and Liquids, John Wiley and Sons, New York, 1954.

[21] C. Wilke, J. Chem. Phys. 18 (4) (1950) 517-519.

[22] S. Lapointe, B. Savard, G. Blanquart, Combust. Flame 162 (9) (2015) 3341-3355.

[23] A. Eucken, Phys. Z. 14 (8) (1913) 324-333.

[24] S. Mathur, P. Tondon, S. Saxena, Mol. Phys. 12 (6) (1967) 569-579.

[25] R. Bird, W. Stewart, E. Lightfoot, Transport phenomena, John Wiley and Sons, New York, 2007. 


\section{List of figure captions}

Figure 1: Schematic of the experimental setup, side view. Dimensions are given in $\mathrm{m}$.

Figure 2: Left: OH-PLIF image, see frame 8 in Fig. 4. Right: schlieren image taken at the same time.

Figure 3: Flame tip velocity plotted against position in the channel. Obstacle is filled in grey. Vertical solid (dashed) lines indicate flame tip positions for frames 1, 4, 7, $10(2,3,5,6,8,9)$ in Fig. 4.

Figure 4: Left: experimental OH-PLIF images from ten experiments. Right: temperature fields from simulations.

Figure 5: Left: magnitude of vorticity. Right: magnitude of velocity with flame front contour $(T=1500 \mathrm{~K})$, black line.

Figure 6: Conditional means of the fuel consumption rate for the non-unity Le number case at three instants. Solid lines represent conditional means from the 2-D simulations. Dashed lines represent solutions from 1-D laminar flames at the corresponding pressure. All curves are normalized by the peak burning rate in the nonunity Le 1-D laminar flame at $15 \mathrm{kPa}$.

Figure 7: Conditional means of the fuel consumption rate for the unity Le number case at three instants. Solid lines represent conditional means from the 2-D simulations. Dashed lines represent solutions from 1-D laminar flames at the corresponding pressure. All curves are normalized by the peak burning rate in the unity Le 1-D laminar flame at $15 \mathrm{kPa}$.

Figure 8: Speed of the tip of the flame, normalized by the respective laminar flame speed, plotted against position in the channel.

\section{List of table captions}

Table 1: Parameters of the simulations and flame properties. $p_{0}$ is the initial pressure, $T_{u}$ is the initial unburnt temperature, $\phi$ is the equivalence ratio, $S_{L}$ is the laminar flame speed, $l_{F}=\left(T_{b}-T_{u}\right) /|\nabla T|_{\max }$ is the laminar flame thickness, $\Delta x=\Delta y$ is the grid spacing, and $T_{\text {peak }}$ is the temperature of peak fuel consumption in the laminar flame. 